\title{
Big Data Challenges in Biometric Technology
}

\author{
Shafagat Mahmudova
}

\author{
Institute of Information Technologies of ANAS, Baku, Azerbaijan
}

\begin{abstract}
This study is devoted to Big Data problems in biometric technologies. It highlights the importance of the use of Big Data technologies in solution of a wide range of issues facing the national security, law enforcement system and defense authorities. The study provides information about Big Data and advanced cloud technologies in the field of biometric technology.
\end{abstract}

Index Terms: Biometric technologies, big data, large scale, data, cloud technology.

(C) 2016 Published by MECS Publisher. Selection and/or peer review under responsibility of the Research Association of Modern Education and Computer Science.

\section{Introduction}

Big data is used for the processing and collection of large-scale and complex data. Management systems of traditional databases are not capable to manage large volumes of unstructured data. Big data, as a rule, is defined as a combination of large and complex data collections, and used when traditional applications fail to manage databases or to process the data. The contents of Big Data shown in Fig.1.

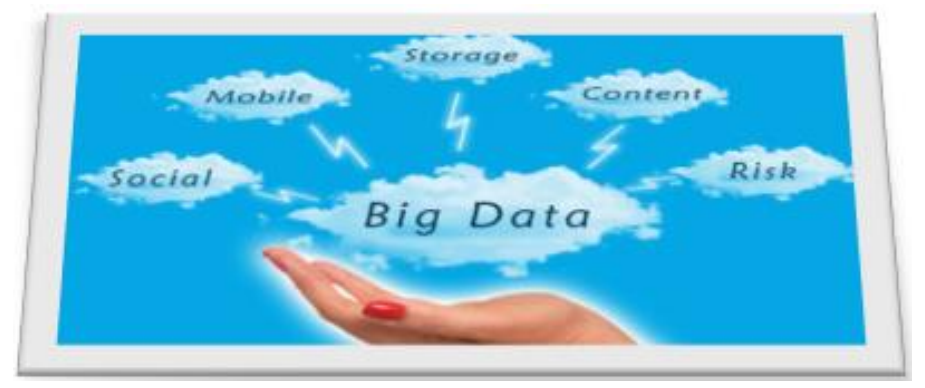

Fig.1. The Contents of Big Data

\footnotetext{
* Corresponding author.
}

E-mail address: 
Big data is very big indeed, and it is a collection of big data massifs, so it cannot process data using traditional calculation methods. Big data includes not only data, but also a variety of technical tools. In comprises the data obtained with the help of various devices and applications [1].

Thus, big data contains three types of high-speed and large volume of data, which are:

- Structured data: relational data;

- Semi-structured data: XML data;

- Unstructured data: Word, PDF and others.

Big data is really crucial for our lives, and it is one of the world's most important technologies.

Big data analytics is in the scope of Data science. Many public and private organizations began to collect the large volume of various data, so they can contain useful information about the problems. For example, use of big data technologies in national intelligence, cyber-security, marketing and medical informatics is of great importance now [2]. Data Science and Big Data shown in Fig. 2.
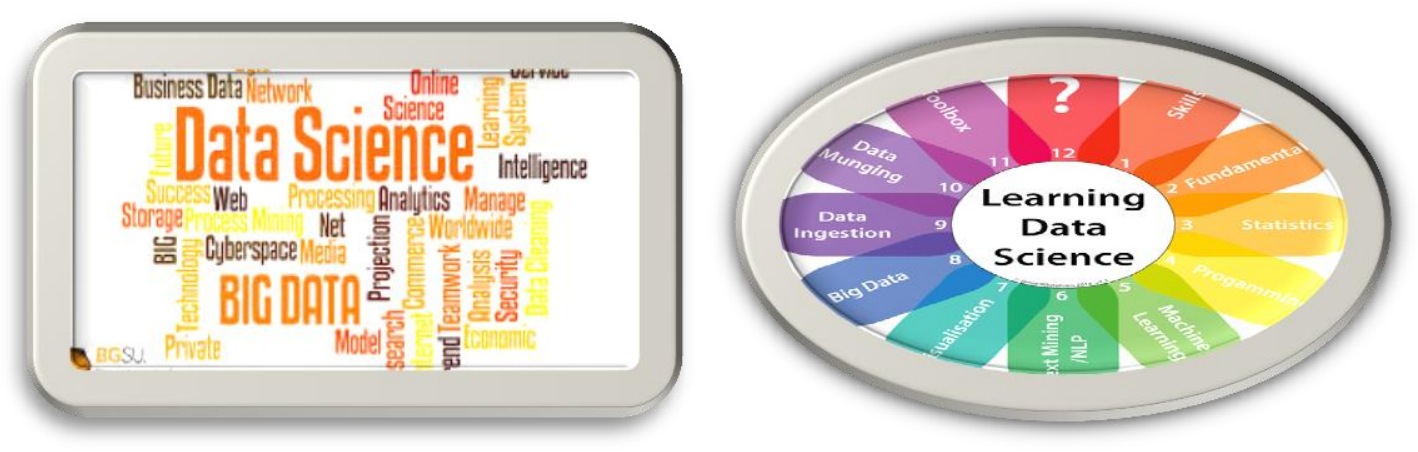

Fig.2. Data Science and Big Data

Through the use of Big Data technologies, Japanese developers began to apply the software to recognize the persons in the black list of buyers of some stores, who were identified as thieves or "complaints".

The police in Tampa, Florida State use Big Data technology called Superbowl XXXV software to identify criminals basing on the scanned images of the face.

Universities and airports in the United States also use Big Data in the field of biometric technologies for the identification of people. Recognition with Big Data technologies shown in Fig. 3.
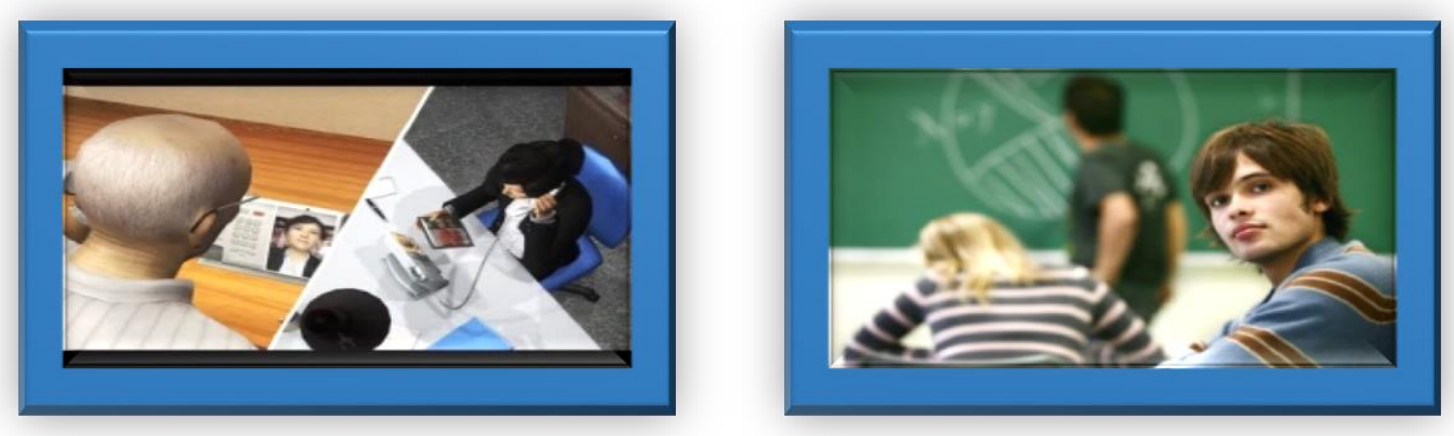


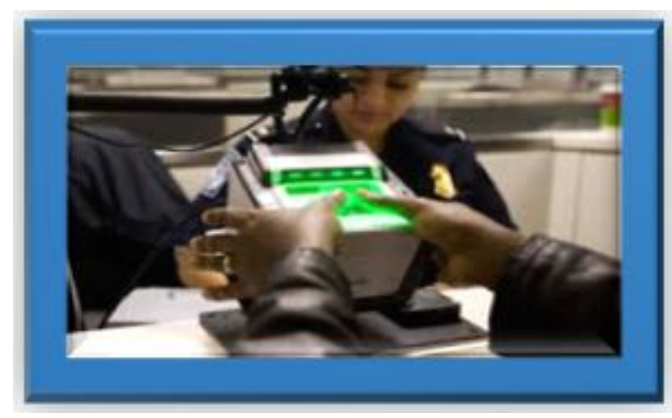

Fig.3. Recognition with Big Data Technologies

Lambda Labs uses its Big Data technology used for face recognition software for Google Glass. Lambda Labs is specialized in artificial intelligence and machine training, so that it processes millions of images of each month. Lambda Labs is designed to solve the complex problems of machine training. Lambda Labs interfaces detect the person and provide web-applications to identify them [3]. Recognition person with software Lambda Labs shown in Fig. 4.
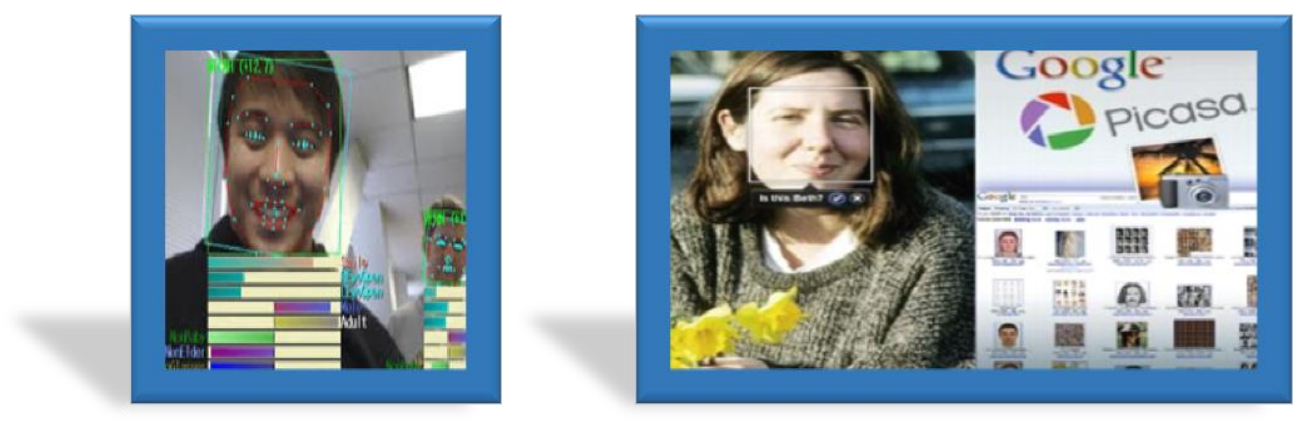

Fig.4. Recognition Person with Software Lambda Labs

Using Face Recognition and Big Data Analysis law enforcement agencies improve their work efficiency related to recognition.

The use of biometric technologies ensuring the security of various identification systems is indispensable.

Information Security [4] as a whole covers a wide range of technologies. The purpose of the teaching of information security at the University of Economics and Finance, to make students master the technology of information security and the method in practical applications.

The advantage of the implementation of all identification algorithms on various biometric feature son a single platform is that the identification results on all features are given to the operator in a single form. Some approaches to results transfer, their assessment and decision-making are designed to use Big Data technologies in the organization of biometric identification algorithms.

Obviously, photos, audio, video data capture large computer memory space and cause big troubles for their processing. Now ordinary servers fail to handle them. In this case, Big Data technologies are required.

As in any other fields, Big Data technologies have some shortcomings regarding risks and confidentiality.

\section{Biometric and Big Data Technology}

Biometric data in the internal affairs agencies, banks and databases of criminal law are quite messy and 
mainly local. This causes certain difficulties for regional fight against crime. It also restricts the search opportunities in detecting suspects, that is, it ends before implementing the identification process. Big Data for biometrics shown in Fig. 5.

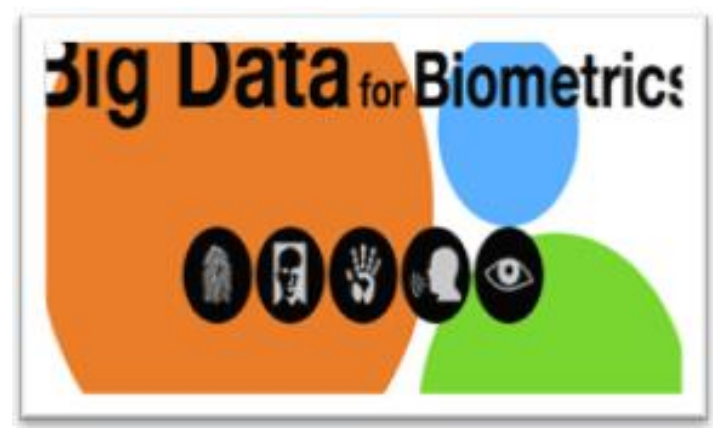

Fig.5. Big Data for Biometrics

Wide use of biometric Big Data technologies is able to solve these problems [5].

Biometric technologies, such as authentication, Biocryptography and Cloud-Based Architecture are ideal for security issues. SaaS, Tygart, MXSERVER and other scan be sited as an example of software [6]. Recognition technology based Tygart shown in Fig. 6.

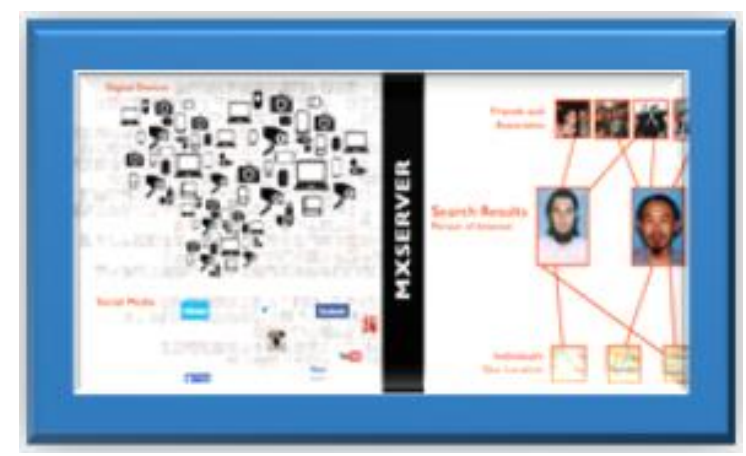

Fig.6. Recognition Technology Based Tygart

Web applications are developed through Software as a service (SaaS) and managed independently [7].

MXSERVER is a system identifying a person based on a powerful server, and used for large-volume videos, photo collections and so on.

Tygart technology-based software is able to group appropriate sections of texts, video, and photos according to the interests of people and delete them [8].

Numerous seminars and conferences have been devoted to Big Data problems in Biometric technologies.

Big Data Biometrics Symposium held in Washington in June 2014 was of great interest. The symposium discussed some problems in the field of Biometric technologies, some of which are:

- Developing major biometric programs and improved standards for big data analysis of countries in the future;

- Compact management of biometric data and finding ways to handle them;

- Future contribution of large-scale analysts to big data and the role of "cloud" technologies in this; 
- Biometric big data analysis and achieving the progress in identification and authentication solutions in a mobile environment to solve the tasks;

- Defining the identification prospects on request using Big Data;

- Studying solutions ways of the problems arising from the use of Big Data in biometric technology, and reducing errors, and so on. [9].

\section{Biometric, Big Data and Cloud Technologies}

Cloud is a new information technology providing single-point access to the distributed resources on request.

The features of these technologies include the ability of the users to work independently, and the accessibility of the cloud anytime, through anywhere and any device (smart phone, laptop, tablet, notebook, and etc.). In addition, the resource sets are introduced to users as a service menu, providing the user to reduce or increase the volume of resources freely, which leads to the widespread use of this technology.

The use of cloud in biometric technologies reduces security and confidentiality problems significantly and helps to prevent any unpleasant incidents as soon as possible. Big Data and Cloud shown in Fig. 7.

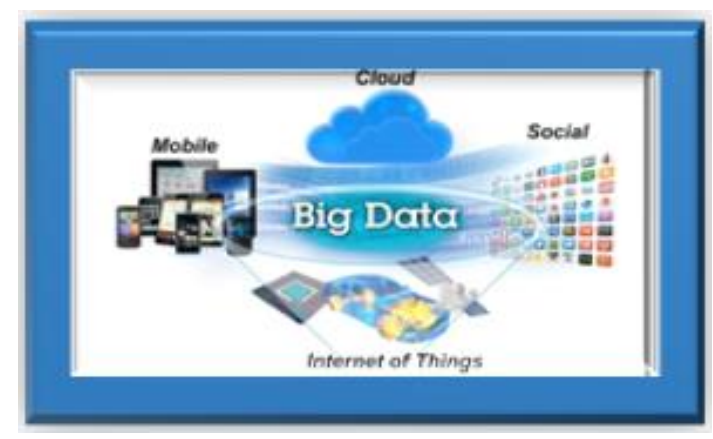

Fig.7. Big Data and Cloud

MXSERVER TM server system processes large-scale video and photo collections using the files obtained through the computers, mobile phones, SIM cards and the video surveillance systems (Fig. 8).

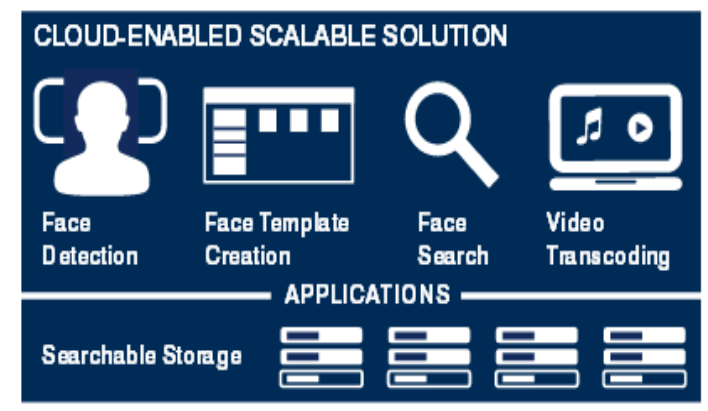

Fig.8. MXSERVER TM Video and Photo Analysis System

Significant increase observed in recognition is the key priority of big data analysis in biometric technologies.

Unlike other forms of biometric technologies, the recognition program can be managed remotely. It can scan photos or videos on the Internet, which is used to mark the photos of people in Facebook or Google.

Big Data technologies include Apache Hive, Apache Giraph, Horton, Hadoop and so on. High-quality 
recognition may be achieved through the use of these technologies (Fig.9).

Apache Hive big data software facilitates the management of the surveys and large-scale data in distributed memory [10].

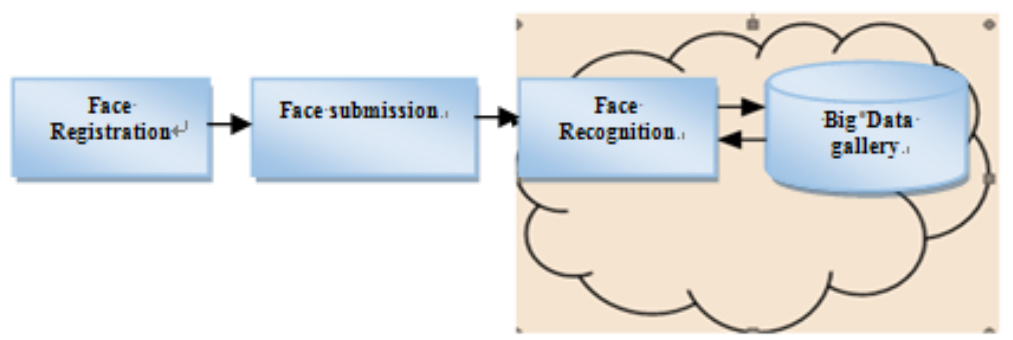

Fig.9. Recognition of Person Through the Use of Cloud and Big Data Technologies

- Face registration;

- Face submission;

- Face recognition;

- Big Data gallery.

The fig. 9 shows that, human face recognition process is implemented as follows.

A new human face submitted to the system through the software is recorded, coded and transfer to cloud; it is processed by the face recognition algorithm, which is provided in advance; it consists of several stages (facial identification, etc.). In the next stage, the human face is compared to the other images in the gallery and the recognition process is implemented.

Apache Giraph is an Apache project designed for graphics processing in large-scale data. Giraph uses Apache Hadoop Map Reduce realization for graphics processing. For example, Facebook uses Giraph to improve the efficiency of images processing [9].

Hadoop is considered to be one of the fundamental big data technologies. Many Hadoop-related projects and technologies soon became independent [11].

Hadoop Distributed File System (HDFS) is designed for storing large-volume distributed files. Hadoop Map Reduce is a software framework for distributed computing programming within Map Reduce paradigm.

Court experts, investigators and security services are making use of MXSERVER (Fig. 10).

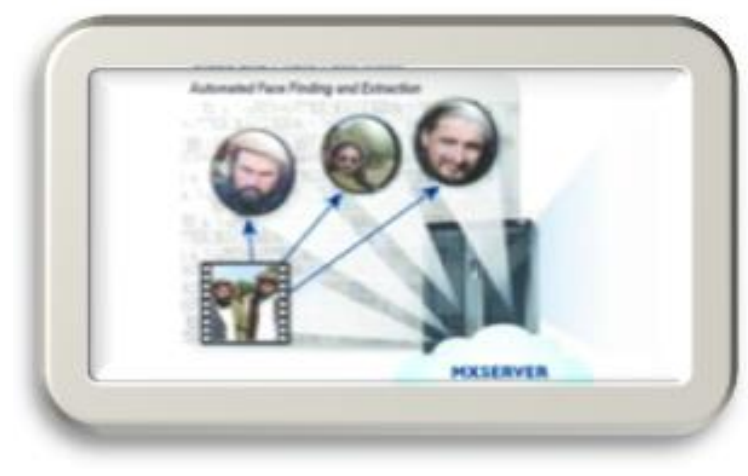

Fig.10. Recognition of a Person Through the use of MXSERVER Technology 
Cloud technology facilitates high scalability, and the system guarantees high-level service for extensive users.

There are many reliable mechanisms in this area, for example, Animetrics system under Cloud-based FR, BioID and Robust Face technology [12, 13].

Data. IT is capable to solve problems of the use of traditional statistical analysis methods of biometric data easily [14].

These associations, among others, are after comes of the ways biometrics permit and embolden more intensive commoditization of physical data. In "When Biometrics Fail: Gender, Race and the Technology of Identity," social scientist Shoshana Magnet writes, "The flimsy material body is rendered rugged as biometric technologies make bodies replicable, transmittable and segment able — breaking the body down into its component parts (from retina to fingerprint) in ways that allow it to be marketed more easily in the transnational marketplace." We've learned about the data economy, but how about the face economy or iris economy or gait economy?

There are entire cooperative sectors which are keen to mine that data and put it to use in a sufficiently large number of ways. Data brokers construct thorough consumer profiles that provided with biometric templates. Salespeople and store security departments can use biometric emanations to bring up your reputation from the database. Your identity can be affixed to your location, which is better followed as you move through avenues, public squares and department stores. Insurance companies, for example, are hungry for the somatic data provided by personal health and fitness monitoring equipment. Now try to perceive what they and others could do with the knowledge and power provided by varied brands of biometrics [15].

Biometrics: Measurable biological and behavioral characteristics that can be used for automated recognition.

Qualities of good biometrics:

Ubiquitous, Consistent, Detectable, Persistent, Unique (within a range of contingency)

That simple scenario that most of us have encountered is a pretty good illustration of both the advantages and deficiencies of the concurrence of biometrics and big data. The face recognition software that makes those Facebook tagging suggestions possible is part of a larger discipline called biometrics that consisted of fingerprints, retinal scans, and gait recognition, and the field is accelerating fast. Combining those capacities with big data analytics tools lets us to realize who you are just by looking at you-whether you're in a photo on Facebook, a video clip, or simply walking around in the world. Is it good or bad? Both of them [16].

"Concerned about security, companies may soon rely on a wide range of biometrics. Turns out you're even more special than you thought and make no mistake: in this era of heightened corporate security, Big Brother is big business. According to the International Biometric Industry Association, the biometrics market will jump from its year 2000 mark of $\$ 165$ million a year to $\$ 2.5$ billion by the end of the decade" [17].

"While fingerprint recognition systems are currently the most widely available biometric identification technology in the market currently, fingerprints are easy to duplicate and thus there are rising security risks for fingerprint-based solutions.

Increasingly, the financial sector is favoring vein patterns as its biometric identifier. Other technologies of promise include 3D facial recognition and iris recognition" [18].

\section{Conclusion}

GLOBAL's biometrics colleagues are developing advanced 'big data' capabilities to aid criminal investigation and counter-terrorism. Sciometrics President Mark Walch explains.

These technologies work in two modalities:

- First of all, investigative - that is processing latent fingerprints captured at a crime scene or other sensitive sites against a database of suspects;

- Secondly as an "afterburner" to vastly improve the ability to make positive identifications from low quality prints and print fragments [19]. 
Big Data problems in the field of biometric technologies are discussed in the case study. Some aspects of the mutual use of Big Data and cloud technologies in biometric technologies are described. We site the use of these technologies in human face recognition as an example.

In near future, the software for Google Glass Will be developed using Big Data technology. The user will be able to display the name and photo of any person passing by in the street on the screen through Facebook (if available) with the help of the application. Currently the company is working on these technologies [20].

In the future, Big Data is expected to be widely used in the field of biometric technologies.

\section{References}

[1] http://www.tutorialspoint.com/hadoop/hadoop_big_data_overview.htm

[2] Najafabadi M, Villanustre F, Khoshgoftaar T, Seliya N, Wald R, Muharemagic E. Deep learning applications and challenges in big data analytics. Journal of Big Data, USA, 2015, vol 2, №1, pp. 1-21.

[3] https://lambdal.com/about

[4] Chunyan Q, Wei Z, Jianhua J, Jialing H. A Teaching Model Application in the Course of Information Security. International Journal of Education and Management Engineering, 2013, 1, pp. 14-20.

[5] Mahmudova Sh. The Role of Biometric Networks in Recognition of Person. Communications, USA, 2015, vol. 3, No. 5, pp. 81-85.

[6] http://findbiometrics.com/face-recognition-and-big-data-analysis-bringing-efficiency-to-lawenforcement/

[7] https://www.pac-online.com/saas-big-data-dynamic-duo-we-have-all-been-waitin

[8] https://www.asdevents.com/event.asp?id=3225\&desc=Biometrics+Big+Data+Symposium

[9] https://hive.apache.org/

[10] https://en.wikipedia.org/wiki/Apache_Giraph

[11] https://ru.wikipedia.org/wiki/Hadoop

[12] Peter P, Bule J, Gros J, Štruc V. Building cloud-based biometric services. Informatica an International Journal of Computing and Informatics, Ljubljanska, 2013, vol 37, No. 1, pp. 115-122.

[13] Vinay A, Vinay S, Rituparna J, Aggrawal T, Balasubramanya K, Natarajan S. Cloud Based Big Data Analytics Framework for Face Recognition in Social Networks using Machine Learning. 2nd International Symposium on Big Data and Cloud Computing (ISBCC'15), VIT University, Chennai, India, Mart 12-13, 2015, vol 50, pp. 623-630.

[14] Sun Y, Chen Y, Wang X, Tang X. Deep learning face representation by joint identification-verification. In Advances in Neural Information Processing Systems, 2014, pp. 1988-1996.

[15] http://america.aljazeera.com/opinions/2014/7/biometrics-big-datamining.html

[16] https://www.researchgate.net/post/Big_data_and_biometric_technologies_

[17] http://ww2.cfo.com/technology/2002/10/the-eyes-have-it/

[18] http://www.electronicsnews.com.au/n...rm=0_fe913f1856-4a2848e020-59145617

[19] http://www.globalgroup.com/newsblog...e-big-data-challenge-in-biometrics/

[20] https://www.linkedin.com/pulse/20140717062548-64875646-why-your-face-matters-more-than-ever 


\section{Authors' Profiles}

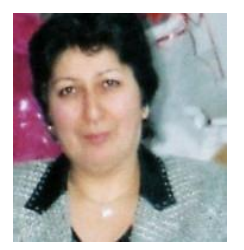

Shafagat Mahmudova, $\mathrm{PhD}$ degree in Technical sciences, Institute of Information Technology of ANAS - Azerbaijan. Currently she conducts research on the "Development of methods and algorithms for the racial identity of human face on the basis of photo-portraits". She teaches at the "Training Innovation Center" of ANAS Institute of Information Technologies. She was elected deputy editor-in-chief of "International Journal of Intelligent Information Processing (IJIIP)", and a member of editorship of "Gconference.NET" portal. She was elected a reviewer of International Journal of Automation and Power Engineering. The journal is published by the Science and Engineering Publishing Company (Riley, Indiana, USA). She was elected a reviewer of "Journal of Control Engineering and Technology (JCET)" and "British Journal of Applied Science \& Technology". Was elected as a member of the International Association for Pattern Recognition (IAPR).

Postal address: AZ1141, Azerbaijan Republic, Baku c., B.Vahabzade str. 9.

How to cite this paper: Shafagat Mahmudova,"Big Data Challenges in Biometric Technology", International Journal of Education and Management Engineering(IJEME), Vol.6, No.5, pp.15-23, 2016.DOI: 10.5815/ijeme.2016.05.02 